\title{
Surgeon Can Contribute to Development of Effective Oral Cancer Control Programs
}

\author{
Kishore Chaudhry ${ }^{1}$ \\ Published online: 20 July 2020 \\ (C) Association of Surgeons of India 2020
}

Presence of an asymptomatic pre-cancerous phase in natural history of oral cancers provides tremendous confidence in screening for its prevention and early detection. But the global evaluations do not recommend screening due to inadequate evidence on its efficacy [1-3]. These evaluations also pointed out limitations in understanding of its natural history, including effectiveness of management of oral potentially malignant disorders (OPMD). High-economy countries do not consider oral cancer a priority, and thus the onus of evaluating the role of screening for oral cancer is on countries like India. Recent global evaluations were largely based on the reports of one Indian randomized controlled trial with 3-yearly cycle of oral cancer screening [4]. This 15 -yearlong study did not show any decrease in incidence rates of the disease but showed an advantage in decrease in mortality rate among high-risk population (tobacco and/or alcohol users). Interestingly, the annual oral cancer screening of Cuba [5] and the 2-yearly screening of Taiwan among tobacco users [6] also failed to demonstrate any reduction in incidence rate, although some advantage in mortality was demonstrated.

To understand this phenomenon, one should revisit the natural history of oral cancer, wherein risk-factors may cause OPMDs that may progress to invasive cancer. The component of disease causation can be divided into a sub-clinical (OPMD and early malignant stage) and a clinical phase (malignant stage). Thus, the sub-clinical stage (when screening can be effective) will have two stages. First is the OPMD stage, wherein the current comprehensive management guidelines aim at their detection, adequate treatment (including complete cessation of tobacco use), and pro-active follow-up for detection of recurrent or new lesions. This action if successful is likely to result in reduction of incidence of oral cancers. This

Kishore Chaudhry

chaudhry_k@hotmail.com; kchaudhry@roseman.edu

1 College of Dental Medicine, Roseman University of Health Sciences, Henderson, NV 89014, USA decreased incidence will automatically result in decreased mortality rate. This is mainly because of the fact that oral cancer screening is unique compared to screening of any other disease. Besides early detection of oral cancer, the major underlying objective of oral cancer screening is primary prevention through detection and treatment of OPMDs. Brief intervention on advice by clinician to quit tobacco is known to be effective $[7,8]$. Invasive malignancy has already occurred during the second stage of sub-clinical phase. Adequately spaced screening cycles can detect these lesions at early stage, where treatment is highly effective and thus prevents mortality. But intervention at this stage will not decrease incidence rate (new cancer cases) of oral cancer, because these cases will enter the cancer registry records. The results from oral cancer screening studies suggest a possible role in the second subclinical stage leading to mortality reduction, but not at the first sub-clinical stage of OPMD. In the absence of screening program, majority of the patients are expected to present during clinical-symptomatic phase, when lower survival and lower quality of life is expected. A large number of tobacco users do present to their surgeon or dentist without visible OPMD. These cases are likely to be labeled as normal and merely receive a nominal advice to quit tobacco.

Thus, the gap in knowledge on causation/progression of oral cancer relates to the OPMD stage. In view of central role of surgery in management of OPMDs, surgeons and dentists can contribute tremendously to the existing knowledge. Compliance to initial (or one-time) participation for examination has been considered as sufficient to suggest the efficacy of screening. But screening is a process with clinical examination just being the first step. To be successful, compliance to referral for adequate treatment is also necessary. The treatment should also not result in recurrence and/or development of new lesions. The Indian oral cancer screening study achieved 92\% participation for examination, but compliance to subsequent steps was low [4]. Not all OPMD patients were selected for biopsy, preference being given for non-homogenous leukoplakia [9]. Less than half of these were treated, and the 
criteria were not standardized. After 12 to 37 months followup of treated OPMDs, $75 \%$ remained disease-free, but characteristics of those developing recurrence or new lesions were not provided. Reasons for non-compliance were also not available. Thus, there is a need to fill gaps in knowledge on these aspects, which can help in determining the exact role of screening in control of oral cancer.

It may not be gainful to perform community-based epidemiological studies for this purpose, due to involved time and cost. Clinic-based studies can accomplish similar results, if planned and analyzed carefully. Such follow-up studies on treated OPMD cases should be pro-active to minimize loss to follow, through telephone or mail contact. Understanding the reasons for recurrence, if the surgery covered the entire atrisk area/depth, assessment of the role of genetic constitution or changes may be just some of the important questions to be answered. How long one needs to follow up before one can be considered as free of OPMD (with or without changes in tobacco habits) is also an important question. Identification of newer and more effective treatment strategies will of course be an area of direct interest for surgeons and dentists.

Surgeons and dental surgeons can surely play a vital role in finding these answers but they will need help from other specialties, like epidemiology, statistics, pathology, genetics, etc. Thus, there is a need to develop inter-disciplinary teams that can identify and answer current dilemmas through carefully planned and unbiasedly interpreted studies.

\section{References}

1. Brocklehurst $P$ et al (2013) Screening programmes for the early detection and prevention of oral cancer. Cochrane Database Syst
Rev (11):CD004150. https://doi.org/10.1002/14651858.CD004150. pub4

2. Lingen MW et al (2017) Evidence-based clinical practice guideline for the evaluation of potentially malignant disorders in the oral cavity: a report of the American Dental Association. J Am Dent Assoc 148(10):712-727.e10

3. Olson CM et al. (2013) U.S. preventive services task force evidence syntheses, formerly systematic evidence reviews, in screening for oral cancer: a targeted evidence update for the U.S. Preventive Services Task Force. Agency for Healthcare Research and Quality (US): Rockville (MD)

4. Sankaranarayanan R, Ramadas K, Thara S, Muwonge R, Thomas G, Anju G, Mathew B (2013) Long term effect of visual screening on oral cancer incidence and mortality in a randomized trial in Kerala, India. Oral Oncol 49(4):314-321

5. Fernandez Garrote L et al (1995) An evaluation of the oral cancer control program in Cuba. Epidemiology 6(4):428-431

6. Chuang SL, Su WWY, Chen SLS, Yen AMF, Wang CP, Fann JCY, Chiu SYH, Lee YC, Chiu HM, Chang DC, Jou YY, Wu CY, Chen HH, Chen MK, Chiou ST (2017) Population-based screening program for reducing oral cancer mortality in 2,334,299 Taiwanese cigarette smokers and/or betel quid chewers. Cancer 123(9):15971609

7. Gupta PC et al (1986) Intervention study for primary prevention of oral cancer among 36000 Indian tobacco users. Lancet 1(8492): 1235-1239

8. Gupta PC, Mehta FS, Pindborg JJ, Bhonsle RB, Murti PR, Daftary DK, Aghi MB (1992) Primary prevention trial of oral cancer in India: a 10-year follow-up study. J Oral Pathol Med 21(10):433-439

9. Pandey M, Thomas G, Somanathan T, Sankaranarayanan R, Abraham EK, Jacob BJ, Mathew B, Trivandrum Oral Cancer Screening Study Group (2001) Evaluation of surgical excision of non-homogeneous oral leukoplakia in a screening intervention trial, Kerala, India. Oral Oncol 37(1):103-109

Publisher's Note Springer Nature remains neutral with regard to jurisdictional claims in published maps and institutional affiliations. 